\title{
Massive Stars: Their Birth Sites and Distribution
}

\author{
Pavel Kroupa ${ }^{\mathrm{a}, \mathrm{b}}$ \\ ${ }^{a}$ Insitut für Theoretische Physik und Astrophysik, Universität Kiel, D-24098 Kiel, \\ Germany \\ ${ }^{\mathrm{b}}$ Heisenberg Fellow
}

\begin{abstract}
The stellar IMF has been found to be an invariant Salpeter power-law $(\alpha=2.35)$ above about $1 M_{\odot}$, but at the same time a massive star typically has more than one low-mass companion. This constrains the possible formation scenarios of massive stars, but also implies that the true, binary-star corrected stellar IMF should be significantly steeper than Salpeter, $\alpha>2.7$. A significant fraction of all OB stars are found relatively far from potential birth sites which is most probably a result of dynamical ejections from cores of binary-rich star clusters. Such cores form rapidly due to dynamical mass segregation, or they are primordial. Probably all OB stars thus form in stellar clusters together with low-mass stars, and they have a rather devastating effect on the embedded cluster by rapidly driving out the remaining gas leaving expanding $\mathrm{OB}$ associations and bound star clusters. The distributed population of OB stars has a measured IMF with $\alpha \approx 4$, which however, does not necessarily constitute a different physical mode for isolated star formation. A steep field-star IMF is obtained naturally because stars form in clusters which are distributed according to a power-law cluster mass function.
\end{abstract}

Key words: massive star, supernovae, star formation, binary and multiple stars, stellar clusters and associations

\section{Introduction}

Stars with masses $m>10 M_{\odot}$ are very much the power engines of galaxies leading brief but brilliant lives. They are the major energy sources for galactic atmospheres and rapidly inject heavy elements into them where they die

Email address: pavel@astrophysik.uni-kiel.de (Pavel Kroupa).

Preprint submitted to New Astronomy Reviews, accepted 7 February 2020 
thus changing galactic weather patterns and thereby playing a major role in regulating star formation. Understanding their distribution, both by mass and spatially, and their birth is therefore of underlying importance for understanding the physical state and chemical content of the interstellar medium and the morphological appearance of galaxies.

This contribution gives an overview of the initial mass function (IMF), of the pairing properties and of the spatial distribution of massive stars, as well as their impact on the birth cloud and survival of star clusters. This subject is also reviewed by Massey (1998, distribution and IMF), Shaerer (2002, IMF), Zinnecker (2003, multiple-star properties) and Garay \& Lizano (1999, environment and formation).

\section{The IMF}

The initial mass function, $\xi(m)$, is the distribution by mass of unevolved, zeroage stars, $d N=\xi(m) d m=\xi_{\mathrm{L}}(m) d \log _{10} m$ being the number of stars in the mass interval $m, m+d m$ and $\log _{10} m, \log _{10} m+d \log _{10} m$, respectively, where $\xi_{\mathrm{L}}(m)=(m \ln 10) \xi(m)$ is the "logarithmic IMF". Inferring masses of stars is in general difficult, because they cannot be weighed. Rather, their luminosity is converted to a mass. This can be tricky through, because optical luminosities do not provide a one-to-one map to the stellar mass, since for example a $4 \mathrm{Myr}$ old, $40 M_{\odot}$-star is brighter with $M_{\mathrm{V}}=-6.6$ than a zero-age, $120 M_{\odot}$ star which has $M_{\mathrm{V}}=-6.2$. Optical photometry is not well suited for measuring the masses of massive stars because they emit most of their radiation in the UV, and spectral classification together with photometry is required (Massey 1998, 2003). If the age, effective temperature, composition and rotational angular momentum vector of a single star are known, then the mass can be calculated given theoretical stellar models. Typically only the luminosity is known rather well, and the effective temperature or spectral type is determined from the star's colour or from spectral analysis. The other quantities are typically not well known and mass estimates are therefore limited to an uncertainty given by the changes in mass induced for changes in compositions and rotation

rates. Stellar-evolution models also need to be improved. For example, Massey (2003) points out that observed red super-giants (RSGs) lie at far too low effective temperatures than are reached by modern evolution models, and that the observed relative numbers of RSGs and WR stars are not reproduced theoretically.

Placing the stars of a population in the HR diagram, their masses can be inferred from a comparison with theoretical stellar evolution tracks. Massey and collaborators have been studying the IMF in OB associations with different metallicities and densities $\lesssim 1 M_{\odot} / \mathrm{pc}^{3}$, and found for $m \gtrsim$ a few $M_{\odot}$ that 
$\xi(m) \propto m^{-\alpha}$ with $\alpha=2.3 \pm 0.1\left(N_{\mathrm{OB}}=1\right.$ association in the Small Magellanic Cloud with metallicity $Z=0.002), \alpha=2.3 \pm 0.1\left(N_{\mathrm{OB}}=10\right.$ in the Large Magellanic Cloud, LMC, with $Z=0.008)$, and $\alpha=2.1 \pm 0.1\left(N_{\mathrm{OB}}=13\right.$ with $Z=0.02$ in the Milky Way, MW). The stellar IMF is thus, to a very good approximation, a Salpeter (1955) power-law, and there is no significant dependence on $Z$ for $Z \gtrsim 0.002$. This rather amazing result is perplexing, because the metallicity defines the cooling rate of a molecular cloud core and thus its fragmentation behaviour, and a dependency of the IMF on $Z$ should therefore exist. Perhaps the dependency will become apparent when young populations with $Z<0.002$ are probed. Also, there does not appear to be a significant dependency on density of the population. The dense $\left(\approx 10^{4} M_{\odot} / \mathrm{pc}^{3}\right)$ and $1-$ 3 Myr old massive star-burst cluster R136 in the 30 Doradus star-forming region in the LMC contains about 39 O3 stars which is more than known to be contained in the rest of the MW, LMC and SMC combined, and again $\alpha=2.35 \pm 0.14$ is found for $m \gtrsim 2.8 M_{\odot}$ (Massey \& Hunter 1998; Massey 1998).

The mass of the most massive star in a population, $m_{\max }$, also appears to be independent of $Z \gtrsim 0.002$ (Massey 1998). This is an important finding because it suggests that radiation pressure on dust grains may not be a significant limiting process during the assembly of massive stars. If the stellar IMF is a Salpeter power-law with a flattening at low masses (see below), then R136, which has a stellar mass of roughly $10^{5} M_{\odot}$, should contain $m>750 M_{\odot}$ stars (provided they can form) and any star cluster more massive than $8000 M_{\odot}$ should contain stars as massive as $200 M_{\odot}$ (Elmegreen 2000; Weidner \& Kroupa 2003). Because stars more massive than $150 M_{\odot}$ have not been found in any population, Weidner \& Kroupa infer that a fundamental maximum stellar mass, $m_{\max *}$, must exist, such that $m_{\max } \leq m_{\max *} \approx 150 M_{\odot}$ independent of the richness of the population. Stars more massive than about $150 M_{\odot}$ cannot form. This conclusion, based on statistics, does not hold true if the stellar IMF were steeper with $\alpha>2.8$ for $m>1 M_{\odot}$, because then stars more massive than about $150 M_{\odot}$ would be too rare to occur even in R136 and even if they can form.

The true stellar IMF (the IMF obtained by counting all individual stars in a population) must be, in fact, significantly steeper than the measured Salpeter value $(\S 3)$. For completeness it should be mentioned that the true stellar IMF is a Salpeter power-law, $\alpha=2.3 \pm 0.3$ in the mass range $0.5 M_{\odot}$-a few $M_{\odot}$. It flattens to $\alpha_{1}=1.3 \pm 0.5$ for $0.08 \leq m / M_{\odot}<0.5$ and even further to $\alpha_{0}=0.3 \pm 0.7$ below $0.08 M_{\odot}$ (Reid et al. 1997; Chabrier 2003; Kroupa 2001, 2002). This IMF is referred to in the following as the "standard IMF". 


\section{$3 \quad$ Multiples}

Defining the companion-star fraction as $C S F=\left(N_{B}+2 N_{T}+3 N_{Q}+\ldots\right) /\left(N_{S}+\right.$ $\left.N_{B}+N_{T}+N_{Q}+\ldots\right)$, where the nominator is the number of companions in $N_{\text {sys }}=N_{S}+N_{B}+N_{T}+N_{Q}+\ldots$ systems or primaries, and $N_{S}, N_{B}, N_{T}, N_{Q}$ is the number of single, binary, triple and quadruple systems, respectively, then massive stars typically have $C S F \approx 1.5$ (Preibisch, Weigelt \& Zinnecker 2001; Zinnecker 2003), while $\mathrm{T}$ Tauri stars have CSF $\approx 1$ (Duchêne 1999) and late-type main-sequence stars typically have $C S F \approx 0.5$.

A particularly well studied population of massive stars is the Orion Nebula Cluster (ONC). The ONC is about $1 \mathrm{Myr}$ old and contains in total between 5000 and $10^{4}$ stars (Hillenbrand 1997; Kroupa 2000), and is still very compact and most probably expanding as a result of very recent gas blow-out (Kroupa, Aarseth \& Hurley 2001). It contains today 27 OB "stars" (Hillenbrand 1997), of which the central Trapezium is composed of the four systems $\theta^{1}$ Ori A,B,C,D. Bispectrum speckle interferometry of 13 OB stars by Preibisch et al. (1999) in the ONC together with additional data shows that the "Trapezium" consists of at least 11 stars with a combined mass of about $100 M_{\odot}$. The low-mass cluster members have $C S F \approx 0.5$ while the $\mathrm{OB}$ stars have a significantly larger $C S F \approx 1.5$. The companion separations of the surveyed systems range from 0.13 to $460 \mathrm{AU}$. Preibisch et al. conclude that on average $\mathrm{O}$ stars have 1.5 to 4 companions, and that the distribution of companions is peaked towards low-mass stars. Duchêne et al. (2001) survey the occurrence of companions within distances of 200-3000 AU around 60 OB stars in the massive young cluster NGC 6611. For mass-ratios larger than $0.1 C S F>1$, and the companion mass-distribution is found to be consistent with random sampling from the IMF. Such systematic and detailed observational surveys are needed for other systems containing massive stars, and Zinnecker (2003) overviews the available results.

The binary-star properties of massive stars are a key constraint on their formation mechanism (next section). But one immediate implication is that the high $C S F$ implies that the true stellar IMF cannot be a Salpeter power-law, because for each massive primary there are typically a few less-massive companions. Detailed corrections to the true $\alpha$ are not available yet, although work is in progress (Weidner \& Kroupa, in preparation). However, a guess can be obtained by noting that for a $100 \%$ binary fraction $(C S F=1)$ and masses picked randomly from the IMF an observed power-law IMF with $\alpha_{\text {obs }}=2.35$ (§ 2) has a true underlying $\alpha \approx 2.7$ (Sagar \& Richtler 1991). Since $C F S>1$ for OB stars, $\alpha>2.7$. 


\section{Clusters and OB associations}

About $50 \%$ of all OB stars are found within associations, and most of the remaining ones are in clusters. It can thus be deduced that most if not all OB stars form in embedded clusters. Assuming a standard IMF with $\alpha=2.35$ or $\alpha=2.7\left(m>1 M_{\odot}\right)$ implies that a single star with a mass of $20 M_{\odot}$ is associated with a cluster of 810 or 3400 stars, respectively. Indeed, observations of rich young clusters show that massive stars and low-mass stars form together; a separate star-formation mode favouring massive stars is not evident (Kroupa 2002). This will be re-addressed in $\S 5$. Low-mass clusters that contain fewer than a few tens of late-type $\mathrm{O}$ stars loose their gas within a cluster-dynamical time once the massive stars "ignite" causing rapid expansion of the stellar system. The binding energy of the embedded cluster, $E_{\mathrm{bin}}=G M_{\mathrm{st}+\mathrm{g}}^{2} / R=9 \times 10^{48} \mathrm{erg}\left(9 \times 10^{50} \mathrm{erg}\right)$ for a star+gas mass of $10^{4} M_{\odot}$ $\left(10^{5} M_{\odot}\right)$ and cluster radius $R=1 \mathrm{pc}$ with, respectively, crossing times (further below) of $0.3 \mathrm{Myr}(0.1 \mathrm{Myr})$. According to the stellar-evolution models of Maeder (1990), a $15 M_{\odot}\left(85 M_{\odot}\right)$ main-sequence star injects $3 \times 10^{50} \mathrm{erg}$ $\left(3 \times 10^{51} \mathrm{erg}\right)$ of radiation and wind energy per $0.1 \mathrm{Myr}$ into the cloud. Selfconsistent radiation-transport hydrodynamics computations such as presented by Freyer, Hensler \& Yorke (2003) are needed to address this issue in detail, but the above estimates suggest that stellar feedback alone can inject enough energy on a time-scale shorter than the dynamical time to unbind the cloud. The resulting evolutionary sequence UC HII region $\longrightarrow$ HII region and the relevant physical processes are described by Garay \& Linzano (1999). A molecular cloud region producing a number of such clusters will thus emerge as an expanding OB association. Massive embedded clusters, such as R136, cannot expel their gas within a dynamical time owing to the depth of the potential well (Goodwin 1997) and consequently survive longer in a denser configuration allowing more time for energy re-distribution among the cluster stars thereby forming bound star clusters that are surrounded by expanding stellar populations (Kroupa \& Boily 2002; Boily \& Kroupa 2003a,b).

Within the clusters, the massive stars are typically centrally concentrated. Examples are the massive R136 cluster, which has been found to be masssegregated (Selman et al. 1999; Bosch et al. 1999) and the low-mass ONC (Hillenbrand 1997), which hosts the Trapezium system at its centre (§3). It is not clear yet if the observed degree of mass segregation in clusters younger than a few Myr is dynamical or primordial in origin. An idealised star cluster consisting of single stars with different masses that is initially in dynamical equilibrium evolves by seeking a new equilibrium state that can never be achieved. An encounter between two unequal stars in the cluster potential imparts kinetic energy to the less-massive star which therefore becomes less bound to the cluster at the expense of the more massive one which consequently sinks towards the potential minimum thereby getting more bound to 
the cluster. As a result it picks-up kinetic energy again and can again exchange its energy with another less-massive star. The process continues until other astrophysical effects such as stellar collisions or mass loss from the cluster core through stellar winds begin to dominate, leaving an expanded low-mass stellar population with an increasingly bound core of massive stars. The timescale for energy equipartition in an idealised cluster has been estimated by Spitzer $\left(1977\right.$, p.74) to be $t_{\mathrm{msegr}} \approx\left(m_{\mathrm{av}} / m_{\mathrm{m}}\right) t_{\mathrm{relax}}$. Here $m_{\mathrm{av}}$ is the average stellar mass $\left(m_{\mathrm{av}} \approx 0.4 M_{\odot}\right)$ and $m_{\mathrm{m}}$ is the mass of the massive star taken to be $m_{\mathrm{m}}=20 M_{\odot}$ for the examples below. The relaxation time, which is the time for significant energy redistribution to occur in a cluster (e.g. Binney \& Tremaine (1987), $t_{\text {relax }} \approx(N / 8 \ln N) t_{\text {cross }}$, and $t_{\text {cross }}=2 R / \sigma$ is the crossing time of a typical star through the cluster which has a characteristic radius $R$ and a velocity dispersion $\sigma=(G M / R)^{0.5}$, where $G=0.0045 \mathrm{pc}^{3} /\left(M_{\odot} \mathrm{Myr}^{2}\right)$ is the gravitational constant. For example, for $\mathrm{R} 136$ with $M \approx 10^{5} M_{\odot}$ and $R \approx 1 \mathrm{pc}$ $t_{\text {cross }} \approx 0.1 \mathrm{Myr}, t_{\text {relax }} \approx 250 \mathrm{Myr}$ and $t_{\text {msegr }} \approx 5 \mathrm{Myr}$, while for the pre-gasexpulsion $\mathrm{ONC}$ with $M_{\mathrm{st}+\mathrm{g}}=10^{4} M_{\odot}$ and $R \approx 0.4 \mathrm{pc}, t_{\text {cross }} \approx 0.08 \mathrm{Myr}$, $t_{\text {relax }} \approx 11 \mathrm{Myr}\left(10^{4}\right.$ stars $)$ and $t_{\mathrm{msegr}} \approx 0.2 \mathrm{Myr}$. Thus, in both cases the equipartition time-scale is comparable to the age of the clusters precluding firm conclusions on the nature of the observed mass segregation. The estimate $t_{\text {msegr }}$ is very crude because once the massive stars dominate the central region there will not be enough low-mass stars to carry away the energy and the process must slow. Self-consistent stellar-dynamical calculations are needed to address this problem in detail. Bonnell \& Davies (1998) argue that the $\mathrm{ONC}$ is too young for mass segregation to have been able to proceed sufficiently far to account for the observed signature. Their modelling relies on using a force-softened stellar-dynamical code to solve the equations of motion, and assumes all stars to be single. Applying a direct summation code with force regularisation, thus avoiding the need for force softening, and assuming a high binary fraction and more massive and denser initial (pre-gas-expulsion) cluster models, Kroupa et al. (2001) found mass segregation to proceed sufficiently far to perhaps account for the observed mass segregation in the densest model of the ONC (Kroupa 2002). This model has an initial central density of $10^{5.8} \mathrm{stars} / \mathrm{pc}^{3}$.

Knowing if the observed mass segregation is primordial is important for constraining formation-theories of massive stars. Radiation pressure on infalling gas with an inter-stellar dust content is sufficient to halt mass accretion for stars more massive than about $10 M_{\odot}$ unless the accretion rate surpasses $10^{-3} M_{\odot} /$ yr and the dust abundance is suppressed (Wolfire \& Cassinelli 1987), and Bonnell, Bate \& Zinnecker (1998) suggest that collisions between intermediate-mass proto-stars in accreting cluster cores may lead to runaway collisional behaviour as the central potential deepens and more low-angular momentum gas is accreted causing further core contraction and so on. This model naturally leads to a high $C S F$ for massive stars, since not all the proto-stars will merge, especially so since during the final stage gas infall is reversed due 
to the energetic feedback from the central massive star(s), immediately leading to core expansion (Vine \& Bonnell 2003). The accretion-induced protostellar collision scenario is supported by Preibisch et al. (1999) on the basis of the multiple-star properties of the massive stars in the ONC and would also lead to a natural explanation of the observed mass segregation in very young clusters, but it requires very high central densities, $10^{8} \mathrm{stars} / \mathrm{pc}^{3}$. This may be compatible with some ultra-compact HII regions that have dimensions of about 0.1 pc (Zinnecker 2003), but the processes occurring within these remain unreachable observationally. Observational evidence from star-forming cloud regions and thus extremely young systems with ages of a few $0.1 \mathrm{Myr}$ do not appear to support this picture entirely. A pre-cluster cloud region with dimensions of not more than a few pc often shows complex morphology with massive stars spread throughout the volume (e.g. Tieftrunk et al. 1998 for the W3 star-forming region containing more than 10 HII regions within a region spanning 2 pc; Alves \& Homeier 2003 for the W49 region which contains more than $100 \mathrm{O}$ stars, about 30 being within a 6 pc diameter region). In some cases massive disks have been detected around massive proto-stars perpendicular to their outflows (e.g. NGC7538S is an embedded $\approx 40 M_{\odot}$ star with a 30000 AU, $400 M_{\odot}$ rotating disk, Sandell, Wright \& Forster 2003). Accretion from a disk lessens the radiation-pressure problem and allows very massive stars to form because accretion occurs first onto the disk and then onto the protostar in its equatorial plane (Jijina \& Adams 1996; Yorke \& Sonnhalter 2002). Massive molecular outflows are also observed emanating from massive proto-stars, implying that they may form like low-mass stars albeit from much denser and warmer cloud cores (Garay \& Lizano 1999; McKee \& Tan 2003). That massive stars may form through proto-stellar accretion together with cloud fragmentation is supported by Duchêne et al. (2001) on the basis of the wide-binary properties of massive stars in M 16, and would be consistent with the very early scattered distribution of massive proto-stars within forming clusters, but would require dynamical mass segregation to proceed swiftly enough to account for the observed mass segregation in young, postgas-expulsion clusters.

However they form, cores of massive stars are unstable because a small $-N$ system decays in about $N$-crossing times. For example, for the ONC the central Trapezium system has a radius $R_{\mathrm{C}} \approx 0.02 \mathrm{pc}$, mass $M_{\mathrm{C}} \approx 100 M_{\odot}$ so that $t_{\text {cross }, \mathrm{C}} \approx 0.008 \mathrm{Myr}$ and the core with 10 stars should decay in about 0.08 Myr. Since the ONC has an age of about $1 \mathrm{Myr}$, the central Trapezium should have decayed long ago, especially if the pre-gas-expulsion density was higher. Even if we allow 0.5 Myr for the Trapezium to form through dynamical mass segregation, it should have decayed a few times by now. This suggests that the Trapezium should be in the final stages of decay, and that the ONC core may have hosted more massive stars than are observed within the Trapezium today. The other massive stars would have been ejected. The two most massive stars in the ONC, $\theta^{1}$ Ori $\mathrm{C}$ and $\theta^{2}$ Ori $\mathrm{A}$, are found to have large 
proper motions (van Altena et al. 1988) perhaps due to on-going break-up of the cluster core.

\section{Isolated $\mathrm{O}$ stars}

Of all $\mathrm{O}$ stars $10-25 \%$ are runaways with speed $v \gtrsim 40 \mathrm{~km} / \mathrm{s}$, while only $2 \%$ of all B stars and only $0.1-0.2 \%$ of all A stars are runaways. Fast O stars have a binary proportion 2-4 times lower than low-velocity OB stars (Gies \& Bolton 1986). Clearly these data have rather important implications for the chemo-dynamical evolution of galaxies, because they indicate that a sizeable fraction of massive stars explode relatively far from their birth-sites. Given typical birth velocities of massive stars of a few $\mathrm{km} / \mathrm{s}$ it has been argued that they cannot drift, within their short lifetimes, to the positions where they are found. In the LMC a number of O3 stars with life-times as short as $2 \mathrm{Myr}$ are found $100 \mathrm{pc}$ or more from viable birth sites. The existens of such outlying massive stars poses a challenge, and it has been argued that such cases may constitute examples of an exotic birth-mode which produces only one massive star and little else, which may be possible if isolated molecular clouds have a stiff equation of state (Spaans \& Silk 2000; Li, Klessen \& McLow 2003). The IMF of the distributed population of OB stars has been measured $\alpha \approx 4 \pm 0.5$ (Massey 1998), and may indicate a variation of the IMF with star-forming conditions (but see the end of this section). However, the invariance of the stellar IMF among OB associations with different metallicity (§ 2) does not appear to support this possibility.

As concluded in $\S 4$ massive stars prefer to reside in dynamically unstable cluster cores, and violent ejections from these may well explain most if not all of the outliers. The sense of the effect would, qualitatively, give the correct trend with stellar mass, because the most-massive stars would be more prone to violent ejections by virtue of their core-residence than the less massive $\mathrm{B}$ and the even less massive A stars which are not observed to form cluster cores with such high binding energies. The trend of runaways with stellar mass cannot, on the other hand, be obtained if a primary star explodes as a supernova thereby freeing its companion which leaves with the circular (because of tidal circularisation of the short-period orbit prior to the explosion) orbital velocity (a few $100 \mathrm{~km} / \mathrm{s}$, e.g. Tauris \& Takens 1998). The problem with this scenario, which undoubtedly leads to some runaways (Portegies Zwart 2000), is that the mass-ratio distribution of massive binaries ( $(3)$ implies that less-massive companions (B and A stars) ought to be expelled with large velocities more often than massive ones because they are more frequent $(\S 3)$.

In an elegant analysis, Clarke \& Pringle (1992) deduce certain characteristics that the massive stars need to have in order for the statistical properties of the 
observed runaways to be explained as being dynamically ejected stars from cluster cores. They conclude that the massive stars need to form in small groups $(N \lesssim 20)$ containing massive binaries with mass-ratios biased towards unity. These groups need to be severely underrepresented in low-mass stars. Modern binary-star data $(\S 3)$ do not appear to be consistent with these constraints, although for example a deficit of low-mass stars has been verified for the core of the ONC (Hillenbrand 1997). Sophisticated stellar-dynamical calculations need to be performed in order to understand the core processes in detail, and to verify the conjecture of Clarke \& Pringle. Such work has been in progress, and for example Zinnecker (2003) reports numerical experiments of clusters with $(C S F \geq 0)$ and realistic IMFs, whereby the binaries are constructed by randomly pairing stars from the IMF in the mass range $0.01-$ $50 M_{\odot}$ and there is no initial mass segregation. This null hypothesis leads to far too many massive primaries having secondaries with a mass ratio $<0.1$ than are observed, but the massive stars rapidly segregate to the cluster centres, exchange secondaries for more massive ones, and for the binary-rich cases up to $40 \%$ of all stars with $m \geq 8 M_{\odot}$ are found outside twice the tidal radius of their cluster by the time they explode. This thus appears to support the dynamical-ejection scenario for a large fraction of OB runaways, but much more detailed work is necessary for firmer confirmation.

Because binary-binary encounters dominate the production of runaway stars in clusters containing binaries, the fundamental interaction reaction is of the type binary+binary $\longrightarrow$ triple(unstable or stable) $+\operatorname{single}_{\mathrm{ej}}(B+B \longrightarrow$ $T^{\mathrm{u} o r \mathrm{~s}}+S_{\mathrm{ej}}$ ) and if the triple is unstable then $T^{\mathrm{u}} \longrightarrow B+S_{\mathrm{ej}}$ follows within typically a few dynamical times of the unstable triple, although the decay occurs stochastically and cannot be predicted given the chaotic nature of the general 3-body Kepler problem. Leonard (1991) has performed a vast number of $B+B$ scattering experiments for different binary-star mass ratios in order to quantify the reaction cross sections and thus the probability of outcome. The asymptotic ejection velocities that can be reached after an infinite number of $B+B$ events are $v_{\text {ej }} \leq v_{\text {esc }}$ for an ejected low-mass star, where $v_{\text {esc }}$ is the escape velocity from the surface of the most massive star involved in the quadruple reaction, and $v_{\text {ej }} \leq 0.5 v_{\text {esc }}$ for an ejected star with an equal mass as the most massive star in the reaction. For example, $v_{\text {esc }}=1400 \mathrm{~km} / \mathrm{s}$ for a $60 M_{\odot}$ star so that $v_{\mathrm{ej}}=700 \mathrm{~km} / \mathrm{s}$ for another $60 M_{\odot}$ star.

Quantitative demonstration that a given OB star has been ejected is difficult and time-consuming, because its orbit in the Galactic potential needs to be calculated backwards in time in order to identify the possible young cluster of origin. This has been achieved beautifully in a few cases (Hoogerwerf et al. 2001; Allen \& Kinman 2003), but some OB stars appear to be located so far from any star-forming sites that they could not have propagated to their present positions given realistic velocities and birth sites near to the Galactic disk. Nevertheless, careful scrutiny (e.g. Ramspeck, Heber \& Moehler 
2001) typically verifies that ejection is viable in most cases. Extreme ejection velocities may be reached in the reaction $B+b h \longrightarrow(b h+S)+S_{\mathrm{ej}}$, where $S_{\mathrm{ej}}$ can attain a velocity as large as $4000 \mathrm{~km} / \mathrm{s}$ if $b h$ is a $10^{6} M_{\odot}$ black hole (Hills 1988).

Returning to the field-star IMF, Kroupa \& Weidner (2003) show that it must be steeper $\left(\alpha_{\text {field }}>\alpha\right.$ for $\left.m>1 M_{\odot}\right)$ than the true, binary-star-corrected IMF which has $\alpha \gtrsim 2.7$ ( $\S 3$ ). Stars form in embedded clusters most of which disperse within a few 100 Myr and which are distributed according to a cluster mass function which is found to be well represented by a power-law with index $\beta \approx 2.2$ (Lada \& Lada 2003; Hunter et al. 2003). Low-mass clusters contain no massive stars, so that the field-star IMF contains fewer massive stars per low-mass star than the population within an individual well-populated cluster does. The result is that $\alpha_{\text {field }} \approx 3.5$ for $\alpha=2.7$, coming close to the field-star IMF determined by Massey and collaborators (beginning of this section).

\section{Conclusions}

The birth of each massive stars is associated with violent gas-dynamical processes and the emergence of a cluster containing thousands of low-mass stars. The measured initial mass function (IMF) of massive stars in clusters is found to be a universal Salpeter power-law $(\alpha=2.35)$. The maximum stellar mass appears to be near $150 M_{\odot}$ and does not depend on metallicity for $Z \gtrsim 0.002$. The limit near $150 M_{\odot}$ may constitute a physical mass limit, or it may constitute a statistical limit beyond which stars are simply too rare to be found even in the most massive clusters. The latter can only be the case if the true, binary-star corrected IMF has $\alpha>2.7$, which in fact is probably the case because most massive stars are in higher-order multiple systems with a massratio distribution that is consistent with random pairing from the IMF and not with a preference towards similar-mass companions. This may perhaps be a result of the coalescence of intermediate proto-stars in accreting cluster cores, but formation through accretion from a massive, unstable disk may also be a possible formation scenario. The field-star IMF, which is the relevant distribution function for energy feedback and metal deposition on galactic scales, is again steeper than this $\left(\alpha_{\text {field }} \gtrsim 3.3\right)$ because stars form in a distribution of star clusters with different masses. Despite their short lifes, a significant fraction of all massive stars are situated far from potential birth sites. In contrast, intermediate stars do not spread as far, and the reason is most probably that massive stars reside in dynamically unstable binary-rich cores in clusters from which they are expelled rapidly. At this stage there is no conclusive evidence for a special mode of star formation which favours production of massive stars in isolated regions of galactic atmospheres. 
Key issues that await further scrutiny are improvement of stellar models, measurement of the IMF in very-low metallicity regions, quantification of the multiple-star properties in dependence of metallicity, the theory of stellardynamical ejections and the formation of cluster cores through dynamical mass segregation, the detailed physical processes involved in expelling residual gas from an embedded cluster, and its reaction to the rapid mass loss in dependence of cluster mass.

I thank the organising committee for the invitation to present this overview at beautiful Kloster Seeon in Bavaria, and I acknowledge support through grants KR1635/3 and KR1635/4.

\section{References}

Allen, C., Kinman T. D. (2003), in Sixth Pacific Rim Conference on Stellar Astrophysics - A tribute to Helmut, Kluwer Publ., in press (astro-ph/0212272)

Alves, J. and Homeier, N. (2003), Ap.J.L., 589, L45

Binney, J. and Tremaine S. (1987), Galactic Dynamics, Princeton University Press

Boily, C. M. and Kroupa, P. (2003a), M.N.R.A.S., 338, 665

Boily, C. M. and Kroupa, P. (2003b), M.N.R.A.S., 338, 673

Bonnell, I. A. and Davies, M. B. (1998), M.N.R.A.S., 295, 691

Bonnell, I. A., Bate, M. R., and Zinnecker, H. (1998), M.N.R.A.S., 298, 93

Bosch, G., Terlevich, R., Melnick, J., \& Selman, F. (1999), A\&AS, 137, 21

Chabrier, G. (2003), PASP, 115, 763

Clarke, C. J. and Pringle, J. E. (1992), M.N.R.A.S., 255, 423

Duchêne, G. (1999), A\&A, 341, 547

Duchêne, G., Simon, T., Eislöffel, J., Bouvier, J. (2001), A\&̇A, 379, 147

Elmegreen, B. G. (2000), Ap.J., 539, 342

Freyer, T., Hensler, G., Yorke, H. W. (2003), Ap.J., in press (astro-ph/0306541)

Garay, G. and Lizano, S. (1999), PASP, 111, 1049

Gies, D. R. and Bolton, C. T. (1986), Ap.J.S, 61, 419

Goodwin, S. P. (1997), M.N.R.A.S., 284, 785

Hillenbrand, L. A. (1997), AJ, 113, 1733

Hills, J. G. (1988), Nature, 331, 687

Hoogerwerf, R., de Bruijne, J. H. J. and de Zeeuw, P. T. (2001), A\&A, 365, 49

Hunter D. A., Elmegreen B. G., Dupuy T. J. and Mortonson M. (2003) AJ, in press (astro-ph/0306528)

Jijina, J. and Adams, F. C. (1996), Ap.J., 462, 874

Kroupa, P. (2000), New Astronomy, 4, 615

Kroupa, P. (2001), M.N.R.A.S., 322, 231 
Kroupa, P. (2002), Science, 295, 82

Kroupa, P. and Boily, C. M. (2002), M.N.R.A.S., 336, 1188

Kroupa, P. and Weidner C. (2003), Ap.J., in press

Kroupa, P., Aarseth, S., \& Hurley, J. (2001), M.N.R.A.S., 321, 699

Lada, C. J. and Lada E. A. (2003), ARAA, 41, in press (astro-ph/0301540)

Leonard, P. J. T. (1991), AJ, 101, 562

Li, Y., Klessen, R. S. and Mac Low, M. (2003), Ap.J., 592, 975

Maeder, A. (1990), A\&AS, 84, 139

Massey, P. (1998), The Stellar Initial Mass Function (38th Herstmonceux Conference), G. Gilmore, D. Howell (eds), ASP Conference Series, 142, 17

Massey, P. (2003), in May 2003 STScI Symp. "The Local Group as Astrophysical Laboratory", in press (astro-ph/0307531)

Massey, P. and Hunter, D. A. (1998), Ap.J., 493, 180

McKee, C. F. and Tan, J. C. (2003), Ap.J., 585, 850

Reid, I. N., Gizis, J. E., \& Hawley, S. L. (2002), AJ, 124, 2721

Portegies Zwart, S. F. (2000), Ap.J., 544, 437

Preibisch, T., Balega, Y., Hofmann, K., Weigelt, G., \& Zinnecker, H. (1999), New Astronomy, 4, 531

Preibisch, T., Weigelt, G., \& Zinnecker, H. (2001), in IAU Symposium, 200, 69

Ramspeck, M., Heber, U., \& Moehler, S. (2001), A\&A, 378, 907

Sagar, R. \& Richtler, T. (1991), A\& A, 250, 324

Salpeter, E. E. (1955), Ap.J., 121, 161

Sandell, G., Wright, M. and Forster, J. R. (2003), Ap.J.L, 590, L45

Scalo, J. M. (1986), Fundamentals of Cosmic Physics, 11, 1

Schaerer, D. (2002), in IAU Symp. 212, K. A. van der Hucht, A. Herrero, C. Esteban, eds, in press (astro-ph/0208227)

Selman, F., Melnick, J., Bosch, G., and Terlevich, R. (1999), A\&A, 347, 532

Spaans, M., Silk, J., (2000), Ap.J., 538, 115

Spitzer L. (1987), Dynamical Evolution of Globular Clusters, Princeton University Press

Tauris, T. M. and Takens, R. J. (1998), A\&A, 330, 1047

Tieftrunk, A. R., Megeath, S. T., Wilson, T. L., \& Rayner, J. T. (1998), $A \mathscr{G} A$, 336, 991

van Altena, W. F., Lee, J. T., Lee, J.-F., Lu, P. K., and Upgren, A. R. (1988), AJ, 95, 1744

Vine, S. G. and Bonnell, I. A. (2003), M.N.R.A.S., 342, 314

Weidner, C., \& Kroupa, P. (2003), M.N.R.A.S., accepted (astro-ph/0308356)

Wolfire, M. G. and Cassinelli, J. P. (1987), Ap.J., 319, 850

Yorke, H. W. and Sonnhalter, C. (2002), Ap.J., 569, 846

Zinnecker, H. (2003), in IAU Symp. 212, K. A. van der Hucht, A. Herrero, C. Esteban, eds, in press (astro-ph/0301078) 